\title{
ФУГИТИВНОСТЬ $\mathrm{Se}_{2}$ (ГАЗ) В СИСТЕМАХ С БЛАГОРОДНЫМИ МЕТАЛЛАМИ: КРИСТАНЛЕИТ $\mathrm{Ag}_{2} \mathrm{Pd}_{3} \mathrm{Se}_{4}-$ НАУМАННИТ $\mathrm{Ag}_{2} \mathrm{Se}-\beta-\mathrm{PdSe}_{2}$ И ЛЮБЕРОИТ $\mathrm{Pt}_{5} \mathrm{Se}_{4}-$ СУДОВИКОВИТ $\mathrm{PtSe}_{2}$
}

\author{
А. В. Криставчук ${ }^{1}$, А. Вымазалова ${ }^{2}$, Е. Г. Осадчий ${ }^{1}$, И. В. Викентьев ${ }^{3}$,
} Д. А. Чареев ${ }^{1,4,5, *}$, академик РАН Н. С. Бортников ${ }^{3}$

Поступило 28.11.2018 г.

\begin{abstract}
Реакции $6 \mathrm{Ag}_{(\mathrm{cr})}+3 \mathrm{PdSe}_{2(\mathrm{cr})}=2 \mathrm{Ag}_{2} \mathrm{Se}_{(\mathrm{cr})}+\mathrm{Ag}_{2} \mathrm{Pd}_{3} \mathrm{Se}_{4(\mathrm{cr})}$ и $12 \mathrm{Ag}_{(\mathrm{cr})}+5 \mathrm{PtSe}_{2(\mathrm{cr})}=6 \mathrm{Ag}_{2} \mathrm{Se}_{(\mathrm{cr})}+\mathrm{Pt}_{5} \mathrm{Se}_{4(\mathrm{cr})}$ были изучены ЭДС-методом в полностью твёрдофазной гальванической ячейке с серебро-проводящим твёрдым электролитом с общим газовым пространством (Ar при атмосферном давлении). В диапазонах $T=425-648$ К и 501-713 К соответственно были получены зависимости ЭДС от температуры, которые были пересчитаны на фугитивность газообразного Se в зависимости от температуры для нонвариантных равновесий $\mathrm{Ag}_{2} \mathrm{Pd}_{3} \mathrm{Se}_{4}$ (кристанлеит)- $\beta-\mathrm{PdSe}_{2}$ (фаза, при низких температурах переходящая в минерал вербикит) - $\mathrm{Ag}_{2} \mathrm{Se}$ (науманнит) и $\mathrm{Pt}_{5} \mathrm{Se}_{4}$ (любероит)- $\mathrm{PtSe}_{2}$ (судовиковит):

$\lg f \mathrm{Se}_{2 \text { (gas) }}\left(\mathrm{Ag}_{2} \mathrm{Pd}_{3} \mathrm{Se}_{4} / \mathrm{Ag}_{2} \mathrm{Se} / \mathrm{PdSe}_{2}\right)=7,710 \pm 0,050-8,524 \mp 0,026 \cdot(1000 / T)$,

$\lg f \mathrm{Se}_{2 \text { (gas) }}\left(\mathrm{Pt}_{5} \mathrm{Se}_{4} / \mathrm{PtSe}_{2}\right)=7,135 \pm 0,027-12,274 \mp 0,016 \cdot(1000 / T)$.

Ключевые слова: ЭДС-метод, селениды ЭПГ, фугитивность селена, кристанлеит.
\end{abstract}

DOI: https://doi.org/10.31857/S0869-56524856720-725

Термодинамические свойства соединений крайне важны для реконструкции условий минералообразования, но для селенидов благородных металлов данные ограничены [1, 2]. Геохимия Se представляет значительный интерес, поскольку он широко используется в областях Нi-tech и является довольно распространённым элементом, типичным для магматических и постмагматических сульфидных систем [3, 4]. Он обычен в медно-никелевых, меднопорфировых эпитермальных, колчеданных, полиметаллических, урановых месторождениях. В большинстве из них Se встречается в рассеянном виде, но в их рудах также обнаружены селеновые минералы (из них 101 - бескислородные соединения).

Цель нашей работы - определение термодинамических свойств соединений $\mathrm{Se}$, имеющих природные аналоги, по результатам электрохимических экспериментов. Подобно сульфидным равновесиям ассоциации селенидов - индикаторы условий ми-

\footnotetext{
1 Институт экспериментальной минералогии

Российской Академии наук, Черноголовка Московской обл.

2 Чешская геологическая служба,

Прага, Чешская Республика

3 Институт геологии рудных месторождений, петрографии, минералогии и геохимии

Российской Академии наук, Москва

4 Физико-технологический институт

Уральского федерального университета, Екатеринбург

5 Казанский (Приволжскиий) федеральный университет

*E-mail: charlic@mail.ru; chareev@iem.ac.ru
}

нералообразования [5]. Расчёт фугитивности $\mathrm{Se}$ из термодинамических свойств отдельных фаз затруднён отсутствием данных для многих соединений и непостоянством их состава. Способ прямого измерения фугитивности летучих компонентов на линии минеральных равновесий наиболее приемлем. Экспериментальное изучение равновесий кристанлеит $\left(\mathrm{Ag}_{2} \mathrm{Pd}_{3} \mathrm{Se}_{4}\right)$-вербикит $\left(\mathrm{PdSe}_{2}\right)$ - науманнит $\left(\mathrm{Ag}_{2} \mathrm{Se}\right)$ и любероит $\left(\mathrm{Pt}_{5} \mathrm{Se}_{4}\right)$-судовиковит $\left(\mathrm{PtSe}_{2}\right)$ ликвидирует существующие пробелы.

В системе $\mathrm{Ag}-\mathrm{Se}$ существует единственное бинарное соединение - селенид $\mathrm{Au} \mathrm{Ag}_{2} \mathrm{Se}$ (науманнит), образующий две модификации: $\alpha-$ ниже $406 \mathrm{~K}$, $\beta$ - выше 406 К до температуры плавления $1170 \mathrm{~K}$ $[4,6]$. В системе $\mathrm{Ag}-\mathrm{Pd}-\mathrm{Se}-$ трехкомпонентный минерал кристанлеит $\mathrm{Ag}_{2} \mathrm{Pd}_{3} \mathrm{Se}_{4}$ [7] и несколько двухкомпонентных: науманнит $\left(\mathrm{Ag}_{2} \mathrm{Se}\right)$, вербикит $\left(\mathrm{PdSe}_{2}\right)$ и палладсеит $\left(\mathrm{Pd}_{17} \mathrm{Se}_{15}\right)$. Описаны несколько двойных и тройных соединений, которые могут соответствовать новым минеральным видам $[8,9]$. Равновесная ассоциация $\mathrm{Ag}_{2} \mathrm{Pd}_{3} \mathrm{Se}_{4} / \mathrm{PdSe}_{2} / \mathrm{Ag}_{2} \mathrm{Se}$ существует до $430{ }^{\circ} \mathrm{C}$ [9]. Все три фазы считаются стехиометрическими, $\mathrm{PdSe}_{2}$ не содержит $\mathrm{Ag}, \mathrm{Ag}_{2} \mathrm{Se}-\mathrm{Pd}$. Выше $430{ }^{\circ} \mathrm{C}$ ассоциация $\mathrm{Ag}_{2} \mathrm{Pd}_{3} \mathrm{Se}_{4} / \mathrm{PdSe}_{2} / \mathrm{Ag}_{2} \mathrm{Se}$ переходит в ассоциацию $\mathrm{PdSe} 2 / \mathrm{Pd}_{17} \mathrm{Se}_{15} / \mathrm{Ag}_{2} \mathrm{Se}$, в которой соединение $\mathrm{Pd}_{17} \mathrm{Se}_{15}$ содержит до 5 ат.\% $\mathrm{Ag}$. При температуре выше $150{ }^{\circ} \mathrm{C}$ вербикит с моноклинной структурой не существует, а стабильна орторомбическая $\beta$-модификация диселенида Pd [9]. Bep- 
бикит устойчив в узком интервале температур, поэтому термодинамические параметры равновесий моноклинного и орторомбического $\mathrm{PdSe}_{2}$ должны отличаться незначительно.

В системе Pt-Se существуют две фазы [8] и соответствующие им природные аналоги: любероит $\mathrm{Pt}_{5} \mathrm{Se}_{4}$ и судовиковит $\mathrm{PtSe}_{2}$. Они устойчивы выше $1000{ }^{\circ} \mathrm{C}$. Тройная система $\mathrm{Ag}-\mathrm{Pt}-\mathrm{Se}$ не изучена. Наши предварительные данные показали, что в ней от 200 до $600{ }^{\circ} \mathrm{C}$ не существует тройных фаз, а бинарные фазы не растворяют в себе третий компонент; показано существование нонвариантной ассоциации $\mathrm{Ag}_{2} \mathrm{Se} / \mathrm{Pt}_{5} \mathrm{Se}_{4} / \mathrm{PtSe}_{2}$.

\section{ТЕОРЕТИЧЕСКОЕ ОБОСНОВАНИЕ}

Термодинамические свойства кристанлеита, диселенида Pd и науманнита изучены с помощью виртуальной твердофазной реакции

$$
3 \mathrm{PdSe}_{2}+6 \mathrm{Ag}=\mathrm{Ag}_{2} \mathrm{Pd}_{3} \mathrm{Se}_{4}+2 \mathrm{Ag}_{2} \mathrm{Se} .
$$

Реакция (1) реализуется в полностью твердотельной гальванической ячейке

$$
\begin{gathered}
\text { (-) } \mathrm{Pt}|\mathrm{Ag}| \mathrm{AgI} \mid \mathrm{PdSe}_{2}, \\
\mathrm{Ag}_{2} \mathrm{Pd}_{3} \mathrm{Se}_{4}, \mathrm{Ag}_{2} \mathrm{Se} \mid \mathrm{Pt}(+) .
\end{gathered}
$$

Между системой сравнения и системой образца находится твёрдый электролит с проводимостью по $\mathrm{Ag}^{+}$, например $\mathrm{AgI}$, позволяющий провести исследования от 420 до $727 \mathrm{~K}$.

Равновесная ЭДС гальванической ячейки с участием $\mathrm{Ag}_{2} \mathrm{Se}$ может изменяться от 0, когда в системе образца (справа) присутствует металлическое $\mathrm{Ag}$, и фугитивность $\mathrm{Se}\left(f \mathrm{Se}_{2}\right)$ отвечает равновесию $\mathrm{Ag} / \mathrm{Ag}_{2} \mathrm{Se}$ до максимального значения, когда в системе образца присутствует жидкий или кристаллический Se.

ЭДС-ячейки связана со свободной энергией реакции по уравнению

$$
\Delta_{\mathrm{r}} G=-n \mathrm{~F} E,
$$

где $\Delta_{\mathrm{r}} G-$ изменение свободной энергии в ходе реакции; $n=6$ - количество электронов, участвующих в электрохимическом процессе; $\mathrm{F}=96485,309 \mathrm{C} \times$ $\times \mathrm{mol}^{-1}-$ константа Фарадея; $E-$ ЭДС гальванической ячейки в вольтах.

Если неизвестны термодинамические свойства одной из участвовавших фаз, но известны $\Delta_{\mathrm{r}} G$ реакции (1), точные составы всех участвующих фаз и термодинамические свойства оставшихся фаз, то возможно получить энергию образования $\left(\Delta_{\mathrm{f}} G_{T}\right)$ данной фазы.
Если составы фаз строго стехиометричны, то ЭДС-ячейки, энергия реакции и энергии образования участвующих фаз в ячейке (А) связаны уравнением

$$
\begin{array}{r}
\Delta_{\mathrm{r}} G=-6 \mathrm{~F} E=2 \Delta_{\mathrm{f}} G_{T}\left(\mathrm{Ag}_{2} \mathrm{Se}, \mathrm{cr}\right)+ \\
+\Delta_{\mathrm{f}} G_{T}\left(\mathrm{Ag}_{2} \mathrm{Pd}_{3} \mathrm{Se}_{4}, \mathrm{cr}\right)-3 \Delta_{\mathrm{f}} G_{T}\left(\mathrm{PdSe}_{2}, \mathrm{cr}\right)
\end{array}
$$

Температурная зависимость фугитивности газообразного $\mathrm{Se}$ в равновесии с ассоциацией $\mathrm{Ag}_{2} \mathrm{Pd}_{3} \mathrm{Se}_{4} /$ $\mathrm{PdSe} \mathrm{e}_{2} / \mathrm{Ag}_{2} \mathrm{Se}$ определяется реакцией

$$
\begin{aligned}
& 3 \mathrm{PdSe}_{2}(\mathrm{cr})+\mathrm{Ag}_{2} \mathrm{Se}(\mathrm{cr})= \\
= & \mathrm{Ag}_{2} \mathrm{Pd}_{3} \mathrm{Se}_{4}(\mathrm{cr})+3 / 2 \mathrm{Se}_{2}(\mathrm{~g})
\end{aligned}
$$

и может быть рассчитана по уравнению

$$
\begin{gathered}
3 / 2 \Delta_{\mathrm{f}} G_{T}\left(\mathrm{Se}_{2}, \text { gas }\right)+3 / 2 \mathrm{R} T \ln f \mathrm{Se}_{2(\mathrm{gas})}+ \\
+\Delta_{\mathrm{f}} G_{T}\left(\mathrm{Ag}_{2} \mathrm{Pd}_{3} \mathrm{Se}_{4}, \mathrm{cr}\right)=3 \Delta_{\mathrm{f}} G_{T}\left(\mathrm{PdSe}_{2}, \mathrm{cr}\right)+ \\
+\Delta_{\mathrm{f}} G_{T}\left(\mathrm{Ag}_{2} \mathrm{Se}, \mathrm{cr}\right),
\end{gathered}
$$

где $\mathrm{R}-$ газовая постоянная, $T-$ температура в Кельвинах.

Сложение уравнений (3) и (5) приводит к уравнению (6), связывающему ЭДС-ячейки (А) и фугитивность $\mathrm{Se}$ в равновесии $\mathrm{Ag}_{2} \mathrm{Pd}_{3} \mathrm{Se}_{4} / \mathrm{PdSe}_{2} / \mathrm{Ag}_{2} \mathrm{Se}$ :

$$
\begin{gathered}
\mathrm{R} T \ln f \mathrm{Se}_{2(\mathrm{gas})}=2 \Delta_{\mathrm{f}} G_{T}\left(\mathrm{Ag}_{2} \mathrm{Se}, \mathrm{cr}\right)+ \\
+4 \mathrm{~F} E-\Delta_{\mathrm{f}} G_{T}\left(\mathrm{Se}_{2}, \text { gas }\right)
\end{gathered}
$$

или к уравнению

$$
\mathrm{R} T \ln f \mathrm{Se}_{2(\mathrm{gas})}=2 \Delta_{\mathrm{f}} G_{T}\left(\mathrm{Ag}_{2} \mathrm{Se}^{*}, \mathrm{cr}\right)+4 \mathrm{~F} E,
$$

где $\Delta_{\mathrm{f}} G_{T}\left(\mathrm{Ag}_{2} \mathrm{Se}^{*}, \mathrm{cr}\right)$ - энергия образования $\mathrm{Ag}_{2} \mathrm{Se}_{(\mathrm{cr})}$ из $\mathrm{Se}_{2 \text { (gas) }}$ и металлического $\mathrm{Ag}$.

Уравнения (6) и (7) не зависят от термодинамических свойств и состава кристанлеита и вербикита. Другими словами, фугитивность $\mathrm{Se}$ в равновесии c $\mathrm{Ag}_{2} \mathrm{Pd}_{3} \mathrm{Se}_{4} / \mathrm{PdSe}_{2} / \mathrm{Ag}_{2} \mathrm{Se}$ может быть получена напрямую из ЭДС измерений ячейки (А) с использованием термодинамических данных только $\mathrm{Ag}_{2} \mathrm{Se}$.

Равновесная ассоциация любероит $\left(\mathrm{Pt}_{5} \mathrm{Se}_{4}\right)$ - судовиковит $\left(\mathrm{PtSe}_{2}\right)$ - науманнит $\left(\mathrm{Ag}_{2} \mathrm{Se}\right)$ может быть изучена в гальванической ячейке с серебро-проводящим твёрдым электролитом

$$
\begin{gathered}
\text { (-) } \mathrm{C}|\mathrm{Ag}| \mathrm{AgI} \mid \mathrm{Pt}_{5} \mathrm{Se}_{4}, \\
\mathrm{PtSe}_{2}, \mathrm{Ag}_{2} \mathrm{Se} \mid \mathrm{C}(+),
\end{gathered}
$$

в которой реализуется электрохимическая реакция

$$
5 \mathrm{PtSe}_{2}+12 \mathrm{Ag}=\mathrm{Pt}_{5} \mathrm{Se}_{4}+6 \mathrm{Ag}_{2} \mathrm{Se} .
$$

Отсутствие надёжных термодинамических данных для $\mathrm{Pt}_{5} \mathrm{Se}_{4}$ и $\mathrm{PtSe}_{2}$ также не позволяет получить термодинамические свойства отдельных фаз. 
Температурная зависимость фугитивности газообразного $\mathrm{Se}$ в равновесии с $\mathrm{Pt}_{5} \mathrm{Se}_{4} / \mathrm{PtSe}_{2} / \mathrm{Ag}_{2} \mathrm{Se}$ определяется реакцией

$$
5 \mathrm{PtSe}_{2}(\mathrm{cr})=\mathrm{Pd}_{5} \mathrm{Se}_{4}(\mathrm{cr})+3 \mathrm{Se}_{2}(\mathrm{~g}),
$$

т.е. не зависит от присутствия науманнита. Фугитивность $\mathrm{Se}$ в равновесии $\mathrm{Pt}_{5} \mathrm{Se}_{4} / \mathrm{PtSe}_{2}$ рассчитывается по уравнению (7), что не требует знания состава и термодинамических свойств любероита и судовиковита.

Ранее нами аналогичным образом была определена фугитивность $\mathrm{S}$ в равновесиях $\mathrm{Fe}_{1-x} \mathrm{~S} / \mathrm{FeS}_{2}$ (гексагональный пирротин-пирит) и $\mathrm{Fe}_{7} \mathrm{~S}_{8} / \mathrm{FeS}_{2}$ (моноклинный пирротин-пирит) [10], $\mathrm{FeSe} / \mathrm{FeSe}_{2}$ (ачавалит (минерал не подтверждён)-ферроселит) [11]. Из полученных нами данных ЭДС может быть рассчитана $f \mathrm{Se}_{2}$ в равновесии с разными ассоциациями в системе $\mathrm{Ag}-\mathrm{Se}$ и $\mathrm{Ag}-\mathrm{Au}-\mathrm{Se}[12,13]$.

Таким образом, для расчёта $f \mathrm{Se}_{2 \text { (gas) }}$ для равновесий, в состав которых входит селенид $\mathrm{Ag}$, необходимо знание термодинамических свойств $\mathrm{Ag}_{2} \mathrm{Se}$. Энергия образования $\beta$-науманнита из серебра и газообразного $\mathrm{Se}$ была рассчитана нами из комбинации данных, полученных в [12, 14]. Методом ЭДС была исследована реакция образования $\alpha-\mathrm{Ag}_{2} \mathrm{Se}$ из металлического $\mathrm{Ag}$ и твёрдого $\mathrm{Se}$ [12]. Реакция образования $\beta-\mathrm{Ag}_{2} \mathrm{Se}$ исследовалась выше температуры $\alpha-\beta$ перехода при 406-460 К. Реакция образования $\beta-\mathrm{Ag}_{2} \mathrm{Se}$ из металлического $\mathrm{Ag}$ и жидкого Se изучена при 639-824 K [14]. Оба экспериментальных массива были объединены, и из них была вычтена энергия образования газообразного $\mathrm{Se}$ из $\mathrm{Se}$, находящегося в стандартном состоянии (твёрдого при $T<490 \mathrm{~K}$ и жидкого при $T>490 \mathrm{~K}$ ). Данные по $\Delta_{\mathrm{f}} G_{T}$ для $\mathrm{Se}_{2}$ были взяты из [15]. В результате был получен массив данных $\Delta_{\mathrm{f}} G_{T}\left(\beta-\mathrm{Ag}_{2} \mathrm{Se}^{*}\right.$, cr) - энергия образования $\beta-\mathrm{Ag}_{2} \mathrm{Se}$ из металлического $\mathrm{Ag}$ и газообразного $\mathrm{Se}_{2}$ в интервале 406$824 \mathrm{~K}$. Массив был аппроксимирован методом нелинейного взвешенного МНК уравнением а + $+\mathrm{b} T+\mathrm{c} T \ln (T)$ из предположения, что $\Delta_{\mathrm{r}} C_{p}$ постоянна и не равна 0 :

$$
\begin{gathered}
\Delta_{\mathrm{f}} G_{T}\left(\beta-\mathrm{Ag}_{2} \mathrm{Se}^{*}, \mathrm{cr}\right)=-109720 \pm 1310+ \\
+(143,7 \mp 17,1) \cdot T- \\
-(15,364 \pm 2,322) \cdot T \cdot \ln T .
\end{gathered}
$$

Подставляя уравнение (10) в уравнение (7), получаем уравнение (11) для определения фугитивности $\mathrm{Se}_{2}$ (gas) в любых ЭДС-измерениях в гальванических ячейках с $\mathrm{Ag}$-проводящим электролитом и включающих $\beta-\mathrm{Ag}_{2} \mathrm{Se}$ в изучаемом фазовом равновесии:

$$
\begin{gathered}
\lg f_{\mathrm{Se}_{2}}=\frac{20,159 \cdot E-1,605 \cdot T \cdot \ln T-11462,2}{T}+ \\
+15,012,
\end{gathered}
$$

где $E-$ ЭДС в милливольтах, $T-$ температура в Кельвинах.

\section{ЭКСПЕРИМЕНТАЛЬНАЯ ЧАСТЬ}

Синтез образцов выполнялся из элементов в вакуумированных кварцевых ампулах при температуре в соответствии с тройной фазовой диаграммой $\mathrm{Ag}-$ $\mathrm{Pd}-\mathrm{Se}$ [9] и бинарной диаграммой $\mathrm{Pt}-\mathrm{Se}$ [8].

Состав полученных смесей подтверждался методом рентгеновской порошковой дифрактометрии на приборе Bruker D8 ADVANCE ( $\mathrm{CuK}_{\alpha 1}$-излучение, графитовый монохроматор), причём использовали базу данных рентгенограмм PCPDFWIN. Химический состав полученных порошков определяли на сканирующем электронном микроскопе TESCAN Vega II XMU с энергодисперсионной системой микроанализа INCA Energy 450/XT (20 kV).

Система сравнения $(\mathrm{Ag}-99,9 \%)$ и твёрдый электролит, как и система образца, представляли собой таблетки диаметром 6 мм. Их основания полировали до зеркального блеска для улучшения электрического контакта. В качестве твёрдого электролита в обеих ячейках использовали особо чистый AgI. Описание установки для проведения ЭДС-измерений с твёрдыми электролитами присутствует в работах $[10,12,13]$.

Температурная зависимость ЭДС-ячейки (А) была изучена в интервале $425-648$ К. Полученный массив данных (рис. 1) не имеет очевидных изломов, что свидетельствует об отсутствии фазовых переходов первого рода в изучаемом температурном интервале у фаз, участвующих в реакции. Массив отличался от линейного, поэтому был аппроксимирован уравнением $E(T)=\mathrm{a}+\mathrm{b} \cdot T+\mathrm{c} \cdot T \cdot \ln (T)$ :

$$
\begin{gathered}
E(\mathrm{~A}), \mathrm{MB}=189,5 \pm 15,817- \\
\quad-(0,9655 \mp 0,2154) \cdot T+ \\
+(0,1625 \pm 0,0296) \cdot T \cdot \ln T,
\end{gathered}
$$

где $(425<T, \mathrm{~K}<648), k=30$ - количество экспериментальных точек.

Термодинамические свойства кристанлеита $\mathrm{Ag}_{2} \mathrm{Pd}_{3} \mathrm{Se}_{4}$ не могут быть рассчитаны из-за отсутствия на данный момент надёжных данных для $\mathrm{PdSe}_{2}$.

Используя экспериментальные данные, было рассчитано значение $\lg f \mathrm{Se}_{2 \text { (gas) }}$ в равновесии с кристанлеит $\left(\mathrm{Ag}_{2} \mathrm{Pd}_{3} \mathrm{Se}_{4}\right)$ - $\beta$-вербикит $\left(\mathrm{PdSe}_{2}\right)$ - науманнит $\left(\mathrm{Ag}_{2} \mathrm{Se}\right)$ по уравнению (11), рис. 1 . Полученные 


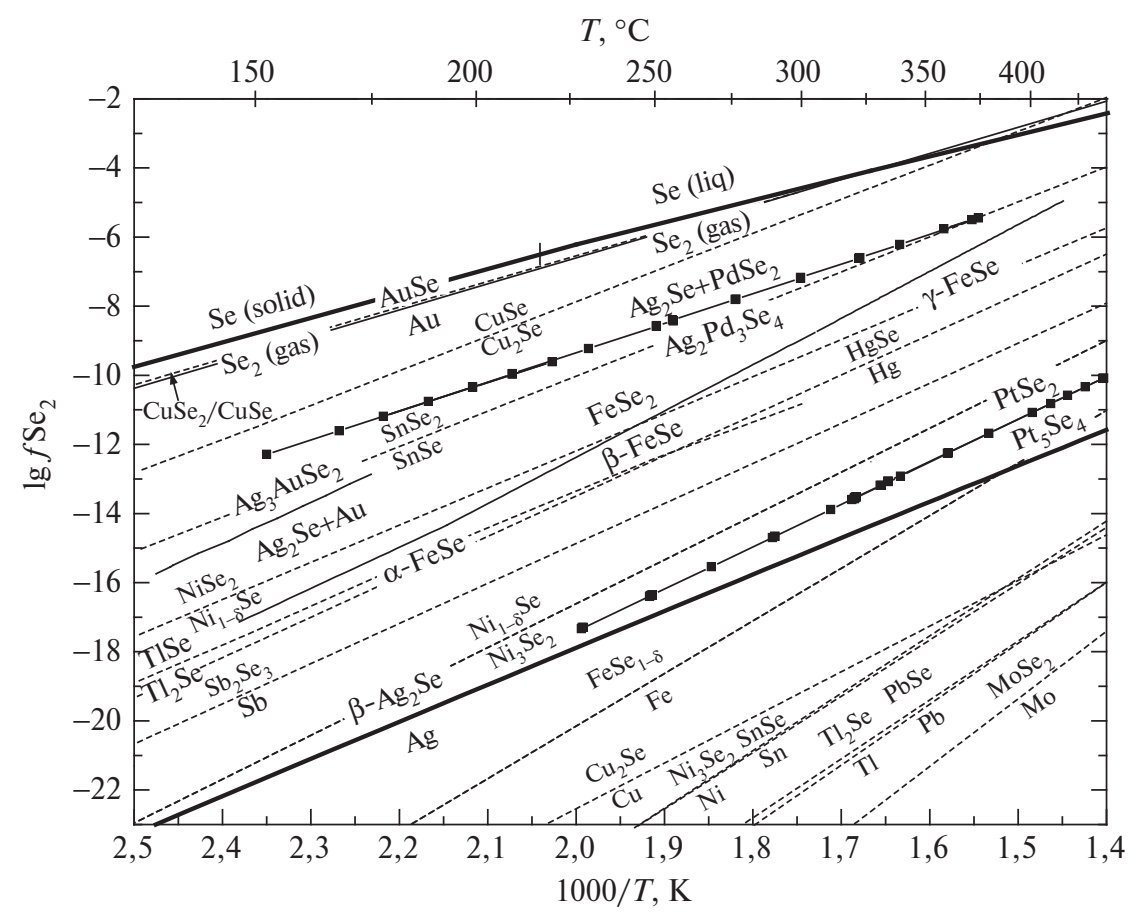

Рис. 1. Зависимость фугитивности газообразного Se от температуры для равновесия кристанлеит $\left(\mathrm{Ag}_{2} \mathrm{Pd}_{3} \mathrm{Se}_{4}\right)-$ $\beta$-вербикит $\left(\mathrm{PdSe}_{2}\right)$ - науманнит $\left(\mathrm{Ag}_{2} \mathrm{Se}\right)$ и для равновесия любероит $\left(\mathrm{Pt}_{5} \mathrm{Se}_{4}\right)$-судовиковит $\left(\mathrm{PtSe}_{2}\right)$. На графике показаны зависимости фугитивности $\mathrm{Se}$ для равновесия $\mathrm{Ag}_{2} \mathrm{Se}$ (науманнит) $/ \mathrm{Ag}$ (данная работа на основании [12, 14]), рассчитанные из (11) при $E=0$, для $\alpha, \beta, \gamma-\mathrm{FeSe}$ (ачавалит) $/ \mathrm{FeSe}_{2}$ (ферроселит) [11], для $\mathrm{AuSe} / \mathrm{Au}$ и $\beta-\mathrm{Ag}_{3} \mathrm{AuSe}_{2}$ (фишессерит) $/ \beta-\mathrm{Ag}_{2} \mathrm{Se} / \mathrm{Au}$, рассчитанные по данным из $[12,13]$. Также показана линия конденсации $\mathrm{Se}$ по данным [15]. Пунктирными линиями и курсивом обозначены равновесия, рассчитанные в [5].

точки лучше всего аппроксимируются линейным уравнением:

$$
\begin{aligned}
& \lg f \mathrm{Se}_{2 \text { (gas) }}\left(\mathrm{Ag}_{2} \mathrm{Pd}_{3} \mathrm{Se}_{4} / \mathrm{Ag}_{2} \mathrm{Se} / \mathrm{PdSe}_{2}\right)= \\
= & 7,710 \pm 0,050-8,524 \mp 0,026 \cdot(1000 / T),
\end{aligned}
$$

где $425<T, \mathrm{~K}<648$.

Температурная зависимость ЭДС-ячейки (В) была изучена в температурном интервале 501-713 K. Результаты измерения зависимости $E(T)$ аппроксимированы логарифмическим уравнением

$$
\begin{gathered}
\mathrm{E}(\mathrm{B}) / \mathrm{MB}=-13,947 \pm 11,142- \\
-(0,721 \mp 0,138) \cdot T+ \\
+(0,1244 \pm 0,0187) \cdot T \cdot \ln T
\end{gathered}
$$

где $501<T, \mathrm{~K}<713$.

Термодинамические свойства одной из фаз с помощью термодинамических свойств другой фазы и энергии Гиббса вычислить на данный момент невозможно, так как отсутствуют данные для $\mathrm{Pt}_{5} \mathrm{Se}_{4}$ и $\mathrm{PtSe}_{2}$. Из данных также была рассчитана $f \mathrm{Se}_{2 \text { (gas) }}$ в равновесии любероит $\left(\mathrm{Pt}_{5} \mathrm{Se}_{4}\right)$ - судовиковит $\left(\mathrm{PtSe}_{2}\right)$, рис. 1. Зависимость ЭДС от температуры нелинейна (14), но величина $\lg f \mathrm{Se}_{2(\mathrm{gas})}$ хорошо аппроксимируется линейным уравнением

$$
\lg f \mathrm{Se}_{2(\mathrm{gas})}\left(\mathrm{Pt}_{5} \mathrm{Se}_{4} / \mathrm{PtSe}_{2}\right)=
$$

$$
\begin{gathered}
=7,135 \pm 0,027-12,274 \mp 0,016 \cdot(1000 / T), \\
501<T, \mathrm{~K}<713 .
\end{gathered}
$$

Кроме двух изученных ассоциаций, на рис. 1 нанесена $f \mathrm{Se}_{2 \text { (gas) }}$ на линии конденсации по данным [15], $f\left(\mathrm{Se}_{2}\right)$ на линии равновесия $\mathrm{Ag}_{2} \mathrm{Se}$ (науманнит) $/ \mathrm{Ag}$, рассчитанная из (11) при $E=0$. Также приведена $f \mathrm{Se}_{2 \text { (gas) }}$ на линии равновесия ачавалитферроселит ( $\left.\mathrm{FeSe} / \mathrm{FeSe}_{2}\right)$ [11], где видны два характерных излома, которые должны быть вызваны $\alpha-\beta$ - и $\beta-\gamma$-фазовыми переходами первого рода в гексагональном $\mathrm{Fe}_{1-\delta} \mathrm{Se}$ (ачавалите). На рис. 1 приведены фугитивности ассоциаций $\beta-\mathrm{Ag}_{3} \mathrm{AuSe}_{2}$ (фишессерит) $/ \beta-\mathrm{Ag}_{2} \mathrm{Se} / \mathrm{Au}$ и $\mathrm{AuSe} / \mathrm{Au}$, рассчитанные по данным из [12, 13]. Фугитивность Se для первой ассоциации была рассчитана из данных ЭДС-измерений с помощью (11). Второй - из термодинамических данных AuSe. Фазовый переход в AuSe при $376{ }^{\circ} \mathrm{C}$ учтён не был. При $T>\sim 300^{\circ} \mathrm{C}$ фугитивность $\mathrm{Se}$ на линии этого равновесия лежит выше кривой конденсации Se. Это согласуется с фазовой диаграммой системы Au-Se [8], так как $>425^{\circ} \mathrm{C}$ AuSe неустойчив и распадается на Au и жидкий Se.

Полученные данные расширяют наши представления по геохимии $\mathrm{Se}$, глубоко изученной лишь для низкотемпературных процессов, а также по гео- 
химии $\mathrm{Ag}, \mathrm{Au}, \mathrm{Pt}, \mathrm{Pd}$; они в комплексе с ранее установленными $P-T-X$-параметрами могут быть использованы для реконструкции условий минералообразования на магматогенных рудных месторождениях.

Источники финансирования. Исследование поддержано РНФ (проект № 14-17-00693-П), Правительством Российской Федерации: Программа 211, соглашение № 02.А03.21.0006 и Программой повышения конкурентоспособности Казанского федерального университета.

\section{СПИСОК ЛИТЕРАТУРЫ}

1. Olin A., Nolang B., Ohman L.-O., Osadchii E.G., Rosen E. Chemical Thermodynamics of Selenium // Chemical Thermodynamics. V. 7. Amsterdam: Elsevier, 2005. P. 894.

2. Vishnevsky A.V., Belogub E.V., Charykova M.V., Krivovichev V.G., Blinov I.A. Thermodynamics of Arsenates, Selenites, and Sulfates in the Oxidation Zone of Sulfide Ores. XIV. Selenium Minerals in the Oxidation Zone of the Yubileynoe Massive Sulfide Deposit, South Urals // Geology of Ore Deposits. 2018. V. 60. № 7. P. 1-9.

3. Иванов В.В., Юшко-Захарова О.Е. Селен. Геологический справочник по сидерофильным и халькофильным редким металлам. М.: Недра, 1989. C. $264-353$.

4. Викентьев И.В., Белогуб Е.В., Молошаг В.П., Еремин Н.И. Селен в колчеданных рудах // ДАН. 2019. T. 484. № 3.

5. Simon G., Essene E.J. Phase Relations Among Selenides, Sulfides, Tellurides, and Oxides; I. Thermodynamic Properties and Calculated Equilibria // Econ. Geol. 1996. V. 91. № 7. Р. 1183-1208.

6. Воронин М.В., Осадчий Е.Г. Определение термодинамических свойств селенида серебра методом гальванической ячейки с твердыми и жидкими электролитами // Электрохимия. 2011. Т. 47. № 4. C. 446-452.

7. Paar W.H., Roberts A.C., Griddle A.J., Topa D. A New Mineral, Chrisstanleyite, $\mathrm{Ag}_{2} \mathrm{Pd}_{3} \mathrm{Se}_{4}$, from Hope's Nose, Torquay, Devon, England // Mineral. Mag. 1998. V. 62. № 2. P. 257-264.

8. Лякишев Н.П. Диаграммы состояния двойных металлических систем: Справочник. М.: Машиностроение, 1997. Т. 1-3. С. 3336.

9. Vymazalová A., Chareev D.A., Kristavchuk A.V., Laufek F., Drábek M. The Ag-Pd-Se System: Phase Relations Involving Minerals and Potential New Minerals // Canad. Mineral. 2014. V. 52. № 1. P. 77-89.

10. Chareev D.A., Voronin M.V., Osadchii E.G. Thermodynamic Study of Monoclinic Pyrrhotite in Equilibrium with Pyrite in the $\mathrm{Ag}-\mathrm{Fe}-\mathrm{S}$ System by SolidState Electrochemical Cell Technique // Amer. Mineral. 2014. V. 99. № 10. P. 2031-2034.

11. Воронин В.М., Чареев Д.А., Осадчий Е.Г. Термодинамические свойства равновесия моно- и диселенида железа при 421-690 К и 1 атм // Материалы 9 Междунар. конф. "Физико-химические и петрофизические исследования в науках о Земле”. М.: ГЕОХИ РАН, 2008. С. 68-70.

12. Osadchii E.G., Echmaeva E.A. The System Ag-Au-Se: Phase Relations below $405 \mathrm{~K}$ and Determination of Standard Thermodynamic Properties of Selenides by Solid-State Galvanic Cell Technique // Amer. Mineral. 2007. V. 92. № 4. P. 640-647.

13. Ечмаева Е.А., Осадчий Е.Г. Определение термодинамических свойств соединений в системах $\mathrm{Ag}-$ $\mathrm{Au}-\mathrm{Se}$ и $\mathrm{Ag}-\mathrm{Au}-\mathrm{Te}$ ЭДС-методом // Геология руд. месторождений. 2009. Т. 51. № 3. С. 276-288.

14. Nasar A., Shamsuddin M. Studies on the Thermodynamic Stability of Silver Selenide // Metallurg. and Materials Trans. B. 1997. V. 28. № 3. P. 519-522.

15. Barin I. Thermochemical Data of Pure Substances. 3rd ed. Weinheim: VCH Publ., 1995. V. 1/2. P. 1885. 


\section{THE $\mathrm{Se}_{2}$ (GAS) FUGACITY IN SYSTEMS WITH NOBLE METALS: \\ CHRISSTANLEYITE $\mathrm{Ag}_{2} \mathrm{Pd}_{3} \mathrm{Se}_{4}$-NAUMANNITE $\mathrm{Ag}_{2} \mathrm{Se}-\beta-\mathrm{PdSe}_{2}$} AND LUBEROITE $\mathrm{Pt}_{5} \mathrm{Se}_{4}-\mathrm{SUDOVIKOVITE} \mathrm{PtSe} \mathrm{P}_{2}$

A. V. Kristavchuk ${ }^{1}$, A. Vymazalova ${ }^{2}$, E. G. Osadchii ${ }^{1}$, I. V. Vikentyev ${ }^{3}$, D. A. Chareev ${ }^{1,4,5}$, Academician of the RAS N. S. Bortnikov ${ }^{3}$

${ }^{1}$ Institute of Experimental Mineralogy, Russian Academy of Sciences, Chernogolovka, Moscow Region, Russian Federation

${ }^{2}$ České geologické služby, Klárov 3, Praha, Czech Republic

${ }^{3}$ Institute of Geology of Ore Deposits, Petrography, Mineralogy, and Geochemistry (IGEM), Russian Academy of Sciences, Moscow, Russian Federation

${ }^{4}$ Institute of Physics and Technology, Yeltsin Ural Federal University, Yekaterinburg, Russian Federation

${ }^{5}$ Kazan (Privolzhskii) Federal University, Kazan, Russian Federation

Received November 28, 2018

The reactions of $6 \mathrm{Ag}_{(\mathrm{cr})}+3 \mathrm{PdSe}_{2(\mathrm{cr})}=2 \mathrm{Ag}_{2} \mathrm{Se}_{(\mathrm{cr})}+\mathrm{Ag}_{2} \mathrm{Pd}_{3} \mathrm{Se}_{4(\mathrm{cr})}$ and $12 \mathrm{Ag}_{(\mathrm{cr})}+5 \mathrm{PtSe}_{2(\mathrm{cr})}=6 \mathrm{Ag}_{2} \mathrm{Se}_{(\mathrm{cr})}+\mathrm{Pt}_{5} \mathrm{Se}_{4(\mathrm{cr})}$ were studied with the EMF method in a completely solid-state galvanic cell with an Ag ion-conducting solid electrolyte with overall gas space (Ar under atmospheric pressure). The EMF vs T dependencies were obtained in the temperature ranges of $T=425-648 \mathrm{~K}$ and $501-713 \mathrm{~K}$, respectively. Then, they were recalculated for gaseous Se fugacity in dependence on the temperature for non-variant equilibriums of $\mathrm{Ag}_{2} \mathrm{Pd}_{3} \mathrm{Se}_{4}$ (chrisstanleyite)- $\beta$ $\mathrm{PdSe}_{2}$ (the phase, which transforms into verbeekite under low temperatures) $-\mathrm{Ag}_{2} \mathrm{Se}$ (naumannite) and $\mathrm{Pt}_{5} \mathrm{Se}_{4}$ (luberoite)- $\mathrm{PtSe}_{2}$ (sudovikovite): $\log f \mathrm{Se}_{2 \text { (gas) }}\left(\mathrm{Ag}_{2} \mathrm{Pd}_{3} \mathrm{Se}_{4} / \mathrm{Ag}_{2} \mathrm{Se} / \mathrm{PdSe}_{2}\right)=7.71 \pm 0.05-8.524 \pm 0.026(1000 / T)$, $\log f \mathrm{Se}_{2 \text { (gas) }}\left(\mathrm{Pt}_{5} \mathrm{Se}_{4} / \mathrm{PtSe}_{2}\right)=7.135 \pm 0.027-12.274 \pm 0.016(1000 / T)$.

Keywords: EMF method, PGE selenides, selenium fugacity, crystallineite. 